\title{
Fracture resistance of palatal cusps defective premolars restored with polyethylene fiber and composite resin
}

\author{
Yanyu MIAO'1, Tiao LIU², Wanwan LEE ${ }^{1}$, Xiuzhi FEI' ${ }^{1}$, Guotao JIANG ${ }^{1}$ and Yuegui JIANG ${ }^{1}$ \\ ${ }^{1}$ Department of Dental Endodontics, School of Dentistry, Xi'an Jiaotong University, Xi'an, China \\ ${ }^{2}$ State Key Laboratory of Military Stomatology, Department of Orthodontics, School of Stomatology, the Fourth Military Medical University, Xi'an, \\ China \\ Corresponding author, Yuegui JIANG; E-mail: jiangyq@mail.xjtu.edu.cn
}

\begin{abstract}
The aim of the study was to compare the fracture resistance of the palatal cusps of defective and endodontically treated premolars after restoration with various restorative techniques. Fifty premolars were assigned to five groups. The first group comprised intact teeth as control (G1), while the other experimental groups (G2, G3, G4 and G5) comprised endodontically-treated teeth, whose palatal cusps were cut and reduced the buccal cusps by $2 \mathrm{~mm}$. G2, composite resin onlay with buccal cusp coverage (CR). G3, CR and fiber post. G4, CR and polyethylene fiber. G5, CR, fiber post and polyethylene fiber. Fracture resistance was tested. The data were recorded in Newton and submitted to ANOVA and Tukey post hoc tests. Statistically significant difference was found between the mean fracture resistances of the groups. Polyethylene fiber reinforced composite restorations strengthened the fracture resistance of palatal cusps defective and endodontically treated premolars.
\end{abstract}

Keywords: Composite resin, Ribbond, Cusp coverage, Fracture mechanics

\section{INTRODUCTION}

Compared to the intact teeth, endodontically treated teeth are considered increasingly susceptible to fracture as they have reduced lower water content, deeper cavities and less dentin ${ }^{1,2}$. The anatomical shape of the maxillary premolar facilitates the fracture of cusps under occlusal loads ${ }^{3}$. Fractures of the teeth palatal cusps command special attention and consideration during the decisionmaking process of their restorative treatment options ${ }^{2)}$.

The remaining structure can be restored with composite, rather than post-core techniques ${ }^{4}$. These attributes of the elastic modulus of composites approximate those of the tooth structure, such that less force will be exerted on the tooth/restoration interface and the stress generated by the occlusal force will be distributed along the tooth ${ }^{5}$. For these reasons, it is possible to restore the premolar without palatal cusp with resin composite. The premolar without palatal cusp can be treated as if only one cavity wall remains, and a post should be inserted ${ }^{6}$. Fiber posts with a modulus of elasticity similar to dentin, when subjected to a compressive load, can better distribute forces along the long axis of the post, which may decrease the probability of root dentin fracture ${ }^{7}$. However, some studies revealed that endodontically treated maxillary premolars without fiber posts exhibited similar fracture resistance to those with a post ${ }^{5,8}$.

Several authors recommend cusp coverage to restore weak posterior teeth ${ }^{9}$. Actually, some clinical trials achieved promising results with this method for the restoration of severally destroyed posterior teeth $^{10,11)}$. However, little data are available about the effect of composite coverage on the fracture resistance of premolar teeth when the palatal cusps are defective.

In recent years, there have been many studies on fiber-reinforced composite (FRC) ${ }^{11,12)}$, and has become a promising alternative for restorative dentistry. The leno wave ultra-high modulus (LWUHM) polyethylene fiber (Ribbond) has provided the opportunity to improve the performance of existing materials. Ribbond is a reinforced ribbon whose special fiber network enables the force distribution along the fiber ${ }^{13)}$, and provides adequate mechanical properties ${ }^{12,13}$. FRC has been used for orthodontic applications and to fabricate single crowns, full and partial coverage-fixed partial dentures, and periodontal splints ${ }^{14}$.

With the development of new materials and technology, new approaches can be implemented when faced with some of these cases to complement traditional methods. Only some studies have investigated the effects of fiber post, direct composite resin or FRC-restored on root-filled treated premolars with MOD cavity ${ }^{15,16)}$. Furthermore, few studies have evaluated the fracture strength of teeth restored with fiber post combined with a fiber ribbon.

The aim of the study was to test the fracture resistance and fracture patterns of endodonticallytreated maxillary premolar teeth with fractured palatal cusps restored by different approaches. The null hypothesis tested was that there is no difference between the different restoration methods used to restore these teeth.

Color figures can be viewed in the online issue, which is available at J-STAGE.

Received Nov 17, 2015: Accepted Feb 3, 2016

doi:10.4012/dmj.2015-394 JOI JST.JSTAGE/dmj/2015-394 


\section{MATERIALS AND METHODS}

Fifty sound, single-rooted, human maxillary premolars, with double canals, freshly-extracted for orthodontic reasons were collected in our study. The debris and soft tissue were carefully removed using a hand scaler. The teeth, free of root canal resorptions, were selected and examined at $10 \times$ magnification using a stereomicroscope (Carl Zeiss, Oberkochen, Germany), and the teeth with cracks were excluded from the study. Next, teeth were stored in a $0.1 \%$ thymol solution at room temperature and used within 3 months. Then, the teeth were classified and randomly distributed, according to the size of the teeth and the tooth root length, into five groups $(n=10)$. The teeth were prepared as follows.

All teeth except those in the G1 were subjected to root canal treatment. Standardized endodontic access cavities were prepared using a water-cooled diamond bur in a high-speed handpiece. A size $15 \mathrm{~K}$-file (Mani, Tochigi, Japan) was inserted into each canal until it could be seen at the apical foramen. The working length was set at $1 \mathrm{~mm}$ short of the apical foramen. The root canals were prepared using the ProTaper system (Dentsply Maillefer, Ballaigues, Switzerland) following the manufacturer's guidelines, from SX to F3 at the working length. The canals were irrigated with $1 \%$ sodium solution and dried with paper points, and then obturated with gutta-percha (Denstply Maillefer) and an AH Plus sealer (Denstply Maillefer) using the cold lateral condensation technique. Subsequently, the access cavities were filled with glass ionomer cement (GIC) (Fuji IX GP, GC, Tokyo, Japan).

After $24 \mathrm{~h}$, the GIC was removed, followed by the removal of the palatal cusps of the experimental groups teeth at the central fissure of the occlusal surface and the gingival wall parallel to cemento-enamel junction (CEJ) using a diamond fissure bur. The buccal cusps were reduced $2 \mathrm{~mm}$. The specimens were subsequently restored with different techniques. The restorative steps for teeth of all the groups are as follows:

In the first group, intact teeth were used as controls.

In the second group, teeth were restored using only composite resin onlay with buccal cusp coverage. The adhesive surfaces were etched with $37 \%$ phosphoric acid (3M ESPE, St. Paul, MN, USA) for $20 \mathrm{~s}$, rinsed with a water spray for $10 \mathrm{~s}$ and gently air dried, leaving the surfaces moist. The surfaces were treated with an adhesive bonding system (Single Bond, 3M ESPE); adhesives were applied in two layers and light-cured for $10 \mathrm{~s}$ with a light-curing unit (VIP Junior, Bisco, Schaumburg, IL, USA) at $600 \mathrm{~mW} / \mathrm{cm}^{2}$ light intensity. After fixation of the matrix band with a retainer, it was restored with a B2-shaded composite resin (Filtek P60, $3 \mathrm{M}$ ESPE) using the incremental technique (restoring first the proximal and lingual wall). The composite resin was applied in $1.5-2 \mathrm{~mm}$ increments and every layer was cured for $20 \mathrm{~s}$ from the occlusal surface. The last layer was light activated for $40 \mathrm{~s}$. Additionally, the reduced buccal cusps were covered to a thickness of 2 $\mathrm{mm}$ with the composite resin. One operator performed all the preparations and restorations.

In the third group, the teeth were restored with glass fiber posts and composite resin. A select universal drill was used to remove $2 / 3$ of the filling material from the root canal, then the post system drill (RelyX Fiber Post drill 2\#) (3M ESPE) was used to widen and shape the root canal following the instructions provided by the manufacturer. Following the post space preparations, the canals were flushed with $2 \mathrm{~mL}$ of $5.25 \% \mathrm{NaOCl}$ and $2 \mathrm{~mL}$ of saline solution. The root canal walls were gently dried, then the resin cements (Rely X Unicem, 3M ESPE) were delivered into the post space. Fiber posts were cleaned with $95 \%$ ethyl alcohol, air-dried, and then seated to canal spaces after the resin cements were dispensed into the post space. Next, using the moderate pressure to hold the post in position for $10 \mathrm{~s}$, the excess of cement was removed with a brush and the material was light-activated for $40 \mathrm{~s}$ from the occlusal face to apical foramen. After cementation, the remaining post length was cut off and the teeth were restored using a procedure similar to that used for the G1.

In the fourth group, the teeth were restored with Ribbond and composite resin as illustrated in Fig. 1. The teeth were restored using the same dentine bonding system and composite resin as those used in G3. Before recovering the reduced buccal cusps and leaving $2 \mathrm{~mm}$ palatal cusps unrestored, the occlusal surface of the teeth between the cusp tips, a 3-mm wide layer of polyethylene ribbon fiber (Ribbond-THM, Ribbond, Seattle, WA, USA) was laid from buccal to lingual direction. The ribbon fiber was then cut, followed by wetting with adhesive resin (Single bond, 3M ESPE), and blotting off the excess with a lint free gauze. The same adhesive system was applied to the cavity walls and cured. Subsequently, a thin 0.5 $\mathrm{mm}$ layer of flowable composite resin (Filtek Supreme Ultra Flowable, 3M ESPE) was applied to the place where ribbon fiber was seated and then uncured. Next, the wetted ribbon fiber was pressed through the flowable composite as close to the cusp as possible and cured for

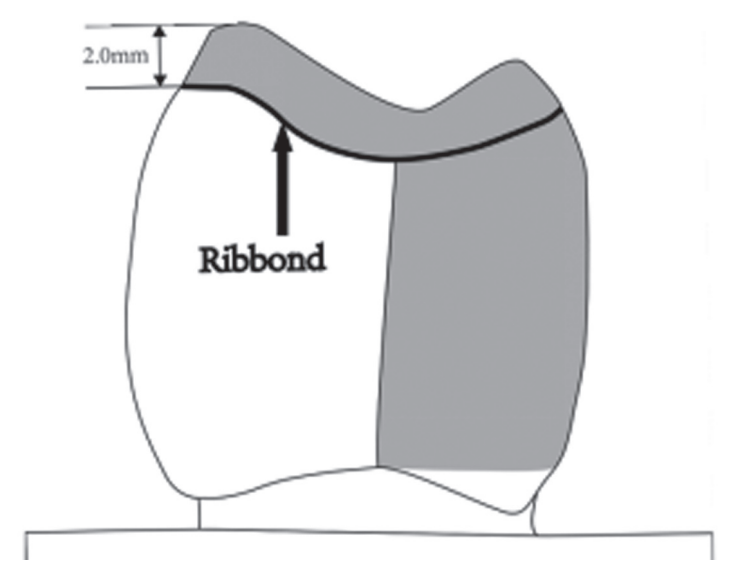

Fig. 1 Appearance of the teeth restored with Ribbond and composite resin (G4). 
40 s. Restoration was recovered with composite, in the same manner as in G2.

In the fifth group, the teeth were restored with glass fiber posts, Ribbond and composite resin. The teeth received a post in a similar manner as described for G3 and were filled with Ribbond and composite resin in a similar way as described for G4.

All the restored and intact teeth were stored at $37^{\circ} \mathrm{C}$ in $100 \%$ humidity for $24 \mathrm{~h}$ and thermocycled for 2,000 cycles at 5 and $55^{\circ} \mathrm{C}$ with a $30 \mathrm{~s}$ dwell time and $5 \mathrm{~s}$ for transfer. Subsequently, all the teeth roots were vertically embedded in self-curing acrylic resin to a level of $1.0 \mathrm{~mm}$ apical to the CEJ and applied about $0.3 \mathrm{~mm}$ thick silicone paste between the roots and acrylic resin to simulate the periodontal ligament.

Static fracture resistance testing was performed with a universal testing machine (Instron, Canton, MA, USA). A vertical compression load test was conducted at a constant crosshead speed of $0.5 \mathrm{~mm} / \mathrm{min}$. The force was applied by a 4 -mm-diameter metal bar, the bar was positioned parallel to the long axes of the teeth, toward the occlusal central fossae of the teeth and contacting the buccal and lingual cusps. The force necessary to fracture the restoration was recorded in Newtons (N). One-way analysis of variance (ANOVA) test followed by Tukey post hoc test were carried out to compare the differences between the five groups at a significance level of 0.05.

Finally, the fractured teeth were examined under 10× magnification to determine the fracture modes. To evaluate whether the fracture mode was favorable (fractures ending less than $1 \mathrm{~mm}$ below the CEJ) or unfavorable (fractures ending more than $1 \mathrm{~mm}$ below the CEJ), a comparison of the failure modes of the specimens was performed using the Chi-square test. All statistical analyses were carried out in SPSS11.0 (SPSS, Chicago, IL, USA).

\section{RESULTS}

The fracture resistance mean and standard deviation values of all samples are presented in Table 1. According to the assessment of the difference between the groups by the ANOVA test the differences between the mean fracture resistance of the groups were statistically significant $(\mathrm{F} 4,50=6.39, p<0.001)$. Therefore our null hypothesis was rejected.
Additionally, the Tukey post hoc test revealed no significant difference between the fracture resistance of the G1, G4 and G5. The G1 was more resistant to fracture than G2 and G3 $(p<0.05)$. In addition, G2, G3 and G4 were found to be statistically similar.

The favorable and unfavorable fractures percentage of the teeth are displayed in Figs. 2 and 3. Favorable fractures were predominant in all specimens (80 to $90 \%$ ) except in G2 and G3. Specifically, in G2, the favorable

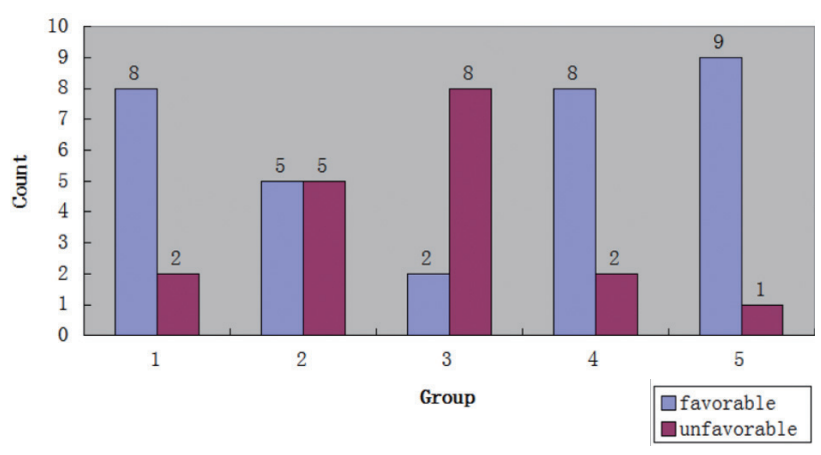

Fig. 2 The number of favorable and unfavorable fracture modes.
A

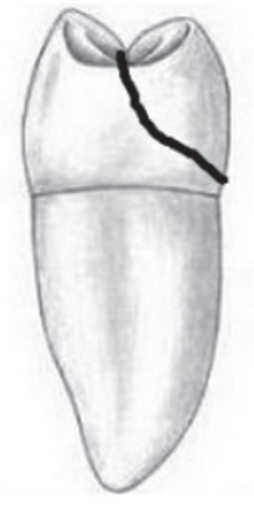

B

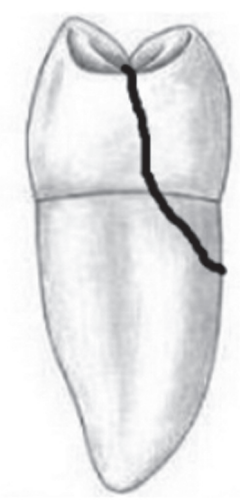

Fig. 3 Mode pattern of 2 types of fracture modes of samples: (A) favorable fracture; (B) unfavorable fracture.

Table 1 Comparison of the fracture strengths between restoration methods

\begin{tabular}{cccc}
\hline Group $(n)$ & Mean (SD) & Subgroups $(\alpha=0.05)$ & - \\
\hline $1(10)$ & $1,333.9(461.1)$ & $\mathrm{A}$ & $\mathrm{B}$ \\
$2(10)$ & $840.6(499.9)$ & - & $\mathrm{B}$ \\
$3(10)$ & $679.1(349.0)$ & - & $\mathrm{B}$ \\
$4(10)$ & $1,036.8(331.8)$ & $\mathrm{A}$ & - \\
$5(10)$ & $1,410.0(266.8)$ & & $\mathrm{A}$ \\
\hline
\end{tabular}


and unfavorable fracture modes were approximately equal, whereas in G3 most of the fractures (80\%) were unfavorable.

\section{DISCUSSION}

It has been demonstrated that normal endodontic access in a healthy tooth has a mere effect on the fracture strength of the tooth ${ }^{17-19)}$. Steele and Johnson reported that the mean fracture strength for unrestored teeth with MOD preparation was 50\% weaker than that of unaltered premolar teeth ${ }^{18)}$. Additionally, an MOD cavity preparation in a root-filled tooth may cause cuspal fracture ${ }^{15,16)}$. As the palatal cusps of teeth fracture, the severely destroyed teeth is usually recommended to be restored by post-core techniques. Metal post as the most commonly used material in clinical practice, but post dislocation and root fracture are the main reasons of failure $^{20)}$. Meanwhile, several attempts have been made to improve the fracture resistance of endodontically treated teeth with different post systems, and could change the rate of root fracture ${ }^{4,8}$. Other studies recommend cusp coverage to restore the fracture resistance $^{9)}$, because they protect cusps which are not shifted to the outside ${ }^{21)}$.

Direct use of composite resins to restore root-filled premolars is deemed to be possible ${ }^{13,22}$. In the present study, cusp coverage with composite did not increase the fracture resistance of premolars compared with intact teeth. Similar results were found in teeth restored with composite onlays ${ }^{23)}$. However, cusp coverage was found to significantly strengthen premolars restored with composite onlays ${ }^{3)}$. This may be due to the axial direction of the compressive load used in these studies, which would lead to different results when compared with the different load degrees along the long axis of the tooth.

In some study, the evaluation of the effect of fiber post and polyethylene fibers on the fracture resistance of the root-filled maxillary premolars revealed that the fiber post and polyethylene fibers (Ribbond) are better able at distributing the forces along the teeth ${ }^{12,24)}$. Consequently, these materials were introduced to conserve the endodontically-treated teeth.

The results of our study did not show significant differences in fracture resistance between the G2 and G3. The finding that the treatment of restored root canal and defective palatal cusps maxillary premolars with a fiber post exhibited a similar fracture resistance to that of those treated only with composite, is consistent with other studies ${ }^{4,25)}$. This could be due to the fact that the tooth crown structure is too little and the resistance to occlusal forces is weak. On the other hand, it has been suggested that the bonding interface of the restorations could disperse functional stresses, which may reinforce weakened tooth structure. Therefore, it seems that even if the fiber post is not placed, it is possible that the bonding area is big enough and could adequately disperse occlusal forces, rendering the tooth strength to confront the loading forces. However, some studies have reported that fiber posts provide better fracture resistance in incisors with indirect veneers ${ }^{26)}$. These contrasting result might be due to the type of restorative material, the type and cavity of the teeth, and the direction of the load applied.

The results of our study showed that the fracture strength of the root-filled teeth restored with Ribbond (G4 and G5) was similar to that of the normal teeth, regardless of the use of the fiber posts. In addition, it has been reported that placing a Ribbond on the occlusal surface could increase the fracture resistance of premolars with MOD cavity $^{13)}$. Another study demonstrated that anterior teeth restored with a Ribbond inside a root canal achieve a higher resistance strength to fracture ${ }^{16)}$. It is thought that combined FRC and dentin as a whole, therefore, if a load force is applied to the sample, the fiber would absorb it and reduce the risk of fracture as the intact tooth ${ }^{12,16)}$. During normal function, the cusps of the opposing tooth exert a wedding effect that pushes the B and L cusps apart. The occlusal forces of the opposing cusp are converted into a lateral force within the composite restoration, which pushes against the $\mathrm{B}$ and $\mathrm{L}$ walls of the cavity. Placing a Ribbond across the cusp B-L splint towards the occlusal forces acts to pull the cusps together when loaded.

The differences between the groups were significant with respect to the failure mode. In the G4 and G5, 80$90 \%$ restorable fracture patterns were observed; these results are similar to those of the G1 (80\% restorable). The structural continuity of the intact teeth has been reserved, so the loading forces applied do not concentrate in special area ${ }^{1)}$. This result is consistent with another study, which confirms that polyethylene fiber has a modifying effect on interfacial stresses ${ }^{13)}$, and the use of FRC restoration can better protect the remaining tooth structure.

The highest percentage of unfavorable fracture mode (80\%) was observed in G3. This might be due to too much root dentin which was cutting, the loading force pass to the weakened root. In addition, the teeth restored using composite with buccal cusp coverage, the composite polymerization shrinkage stresses can impact the failure mode ${ }^{1)}$. Some studies have also shown that the placement of fiber post produces no improvement in the failure mode of maxillary premolars ${ }^{4}$. In G2, 50\% of the fractures were unfavorable, which is higher than those of the intact teeth. When a premolar is restored by full-coverage restorations the stress concentrates on the intercuspal fissure under loading ${ }^{27)}$. It is plausible that restorations with cuspal coverage facilitate fractures.

The loading force was applied at the central fossa with axial direction of the compressive load of the tooth to simulate occlusal force during this study. However, this may be different to clinical conditions where the extensive occlusal loading applied disperses onto the restorations and, therefore, must be evaluated in future experiments. The different loading conditions such as wedges and balls with various diameters, lead to different results in various studies. Therefore, the relevance of the differences between the various research results depend 
on the device and circumstances of the study, rather than the actual values of the fracture loads. Accordingly, the results should be interpreted with caution.

\section{CONCLUSION}

Within the limitations of this laboratory experiment, it may be concluded that regardless of the use of the fiber posts, restorations with ribbon fiber-reinforced resin provided superior fracture resistance of premolars with defective palatal cusps and endodontic access cavities when compared with conventional direct restorative techniques. However, additional long-term clinical studies are required to verify these findings.

\section{ACKNOWLEDGMENTS}

This study was supported in part by Grant No.2012K16-07-03 from the Technologies Research and Development Program of Shanxi China and Grant No. SF1421 (3) from the Science and Technologies Program of Xi'an China.

\section{REFERENCES}

1) Ausiello P, Apicella A, Davidson CL. Effect of adhesive layer properties on stress distribution in composite restorations: a 3D finite element analysis. Dent Mater 2002; 18: 295-303.

2) Goncalves LA, Vansan LP, Paulino. Fracture resistance of weakened roots restored with a transilluminating post and adhesive restorative materials. J Prosthet Dent 2006; 96: 339-344.

3) Mondelli RF, Ishikiriama SK, de Oliveira Filho O, Mondelli J. Fracture resistance of weakened teeth restored with condensable resin with and without cusp coverage. J Appl Oral Sci 2009; 17: 161-165

4) Fokkinga WA, Le Bell AM, Kreulen CM, Lassila LV, Vallittu PK, Creugers NH. Ex vivo fracture resistance of direct resin composite complete crowns with and without posts on maxillary premolars. Int Endod J 2005; 38: 230-237.

5) Scotti N, Scansetti M, Rota R. The effect of the post length and cusp coverage on the cycling and static load of endodontically treated maxillary premolars. Clin Oral Investig 2011; 15: 923-929.

6) Peroz I, Blankenstein F, Lange KP, Naumann M. Restoring endodontically treated teeth with posts and cores - a review. Quintessence Int 2005; 36: 737-746.

7) Salameh Z, Sorrentino R. The Effect of different full-coverage crown systems on fracture resistance and failure pattern of endodontically treated maxillary incisors restored with and without glass fiber posts. J Endod 2008; 34: 842-846.

8) Mohammadi N, Kahnamoii MA, Yeganeh PK. Effect of fiber post and cusp coverage on fracture resistance of endodontically treated maxillary premolars directly restored with composite resin. J Endod 2009; 35: 1428-1432.

9) ElAyouti A, Serry MI. Influence of cusp coverage on the fracture resistance of premolars with endodontic access cavities. Int Endod J 2011; 44: 543-549.

10) Deliperi S, Bardwell DN. Clinical evaluation of direct cuspal coverage with posterior composite resin restorations. J Esthet Restor Dent 2009; 18: 256-265.

11) Deliperia S, Bardwell DN. Reconstruction of devital teeth using direct fiber-reinforced composite resins: a case report. J Adhes Dent 2005; 7: 165-171.

12) Costa S, Silva-Sousa Y, Curylofo F. Fracture resistance of mechanically compromised premolars restored with polyethylene fiber and adhesive materials. Int J Adhes Adhes 2014; 50; 211-215.

13) Sengun A, Cobankara FK, Orucoglu H. Effect of a new restoration technique on fracture resistance of endodontically treated teeth. Dent Traumatol 2008; 24: 214-219.

14) Tuloglu N, Bayrak S, Tunc ES. Different clinical applications of bondable reinforcement ribbond in pediatric dentistry. Eur J Dent 2009; 3: 329-334.

15) Deliperi S. Direct fiber-reinforced composite restoration in an endodontically-treated molar: A three-year case report. Oper Dent 2008; 33: 209-214.

16) Akman S, Akman M, Eskitascioglu G, Belli S. Influence of several fibre-reinforced composite restoration techniques on cusp movement and fracture strength of molar teeth. Int Endod J 2011; 44: 407-415.

17) Reeh ES, Messer HH, Douglas WH. Reduction in tooth stiffness as a result of endodontic and restorative procedures. J Endod 1989; 15: 512-516.

18) Steele A, Johnson BR. In vitro fracture strength of endodontically treated molars. J Endod 1999; 25: 6-8.

19) Bader JD, Shugars DA, Martin JA. Risk indicators for posterior tooth fracture. J Am Dent Assoc 2004; 135: 883-892.

20) Sorensen JA, Martinoff JT. Intracoronal reinforcement and coronal coverage: a study of endodontically treated teeth. J Prosthet Dent 1984; 51: 780-784.

21) Cheung W. A review of the management of endodontically treated teeth: post, core and the final restoration. J Am Dent Assoc 2005; 136: 611-619.

22) Khan SI, Anupama R, Deepalakshmi M, Kumar KS. Effect of two different types of fibers on the fracture resistance of endodontically treated molars restored with composite resin. J Adhes Dent 2013; 15: 167-171.

23) Krejci I, Duc O, Dietschi D, de Campos E. Marginal adaptation, retention and fracture resistance of adhesive composite restorations on devital teeth with and without posts. Oper Dent 2003; 28: 127-135.

24) Kivanç BH, Alaçam T, Gorgul G. Fracture resistance of premolars with one remaining cavity wall restored using different techniques. Dent Mater J 2010; 29: 262-267.

25) Siso ŞH, Hürmüzlü F, Turgut M, Altundaşar E, Serper A, Er K. Fracture resistance of the buccal cusps of root filled maxillary premolar teeth restored with various techniques. Int Endod J 2007; 40: 161-168.

26) D'Arcangelo C, De Angelis F, Vadini M, Zazzeroni S, Ciampoli C, D'Amario M. In vitro fracture resistance and deflection of pulpless teeth restored with fiber posts and prepared for veneers. J Endod 2008; 34: 838-841.

27) Seow LL, Toh CG, Fok AS, Wilson NH. A finite element analysis of ceramic restorations in endodontically treated premolars. Am J Dent 2008; 21: 331-336. 